\section{Guided self-help in primary care mental health}

\author{
Meta-synthesis of qualitative studies of patient experience
}

NAGINA KHAN, PETER BOWER and ANNE ROGERS

\begin{abstract}
Background There is a gap between the supply of trained cognitive-behavioural therapists to treat depression and demand for care in the community. There is interest in the potential of self-help interventions, which require less input from a therapist. However, the design of effective self-help interventions is complex. Qualitative research can help to explore some of this complexity.
\end{abstract}

\begin{abstract}
Aims The study aimed to identify qualitative studies of patient experience of depression management in primary care, synthesise these studies to develop an explanatory framework, and then apply this framework to the development of a guided self-help intervention for depression.
\end{abstract}

\section{Method A meta-synthesis was conducted of published qualitative research.}

\section{Results The synthesis revealed a number of themes, including the nature of personal experience in depression; help- seeking in primary care; control and helplessness in engagement with treatment; stigma associated with treatment; and patients' understandings of self-help interventions.}

Conclusions This meta-synthesis of qualitative studies provided a useful explanatory framework for the development of effective and acceptable guided self-help interventions for depression.

Declaration of interest None. Funding detailed in Acknowledgements.
Cognitive-behavioural therapy is an effective treatment for depression (Department of Health, 2001), but access is characterised by long waiting lists (Lovell et al, 2003). One way of overcoming poor access to this therapy is through the use of 'selfhelp' versions, which are less reliant on the availability of therapists. Self-help involves delivering cognitive-behavioural techniques through a 'health technology' (Richards et al, 2003), such as written materials or information technology (Marrs, 1995; Proudfoot et al, 2004). Although these technologies may be provided alone (so-called 'pure' self-help), they may be facilitated through a small amount of contact with a therapist (a model described as 'guided self-help'). There is evidence that guided self-help is effective (Anderson et $a l, 2005)$ and such interventions provide a potential solution to the gap between demand for cognitive-behavioural therapy and supply, because patients can be treated without the need for large increases in the number of therapists.

\section{METHOD}

Although evidence exists for the effectiveness of guided self-help, this effectiveness varies significantly with different types of guided self-help (Anderson et al, 2005; Mead et al, 2005; Salkovskis et al, 2006), which means that there is significant ambiguity over the optimal design of such an intervention to maximise effectiveness and acceptability. For example, how much guidance should be provided? What is the optimal balance between activities that develop the therapeutic alliance and those focused on teaching cognitive-behavioural techniques? How can the materials be designed to engage patients most effectively? How much reliance should be placed on the agency and experience of the individual patient rather than that of the therapist?
Mental health interventions are increasingly complex, involving a number of different 'active ingredients' to achieve change (Medical Research Council, 2000). Understanding the contexts and ways in which such interventions achieve their effects is crucial for scientific understanding and effective clinical delivery (Campbell $e t$ al, 2000). The 'phased' development of complex interventions has been advocated (Medical Research Council, 2000), and there is interest in the role of qualitative methods alongside randomised controlled trials (Donovan et al, 2002). Qualitative research can help to explore some of this complexity and increase our understanding of the way in which interventions are used and experienced.

The qualitative methodology used in this study was meta-synthesis. The technique has some similarity to quantitative meta-analysis, involving the development of an overview of research, but is based on qualitative papers. Meta-synthesis assists knowledge synthesis through a process of re-conceptualisation of themes across a number of published qualitative studies (Noblit \& Hare, 1988). The method has been applied to particular exemplars in the area of healthcare (Britten et al, 2002; Campbell et al, 2003). The synthesis is derived through the transfer of 'ideas, concepts and metaphors' across different studies (Britten et al, 2002), where the interpretations and explanations in the original studies undergo a process of deconstruction, translation and reconstruction as 'a means to grasp the particulars within the wholes' (Thorne et al, 2004). A distinguishing characteristic of this method is that translations are not literal, but are concerned with the preservation of meaning across studies (Britten et al, 2002). Relationships between studies can be described in a number of ways (Noblit \& Hare, 1988). For the present purposes, a 'line of argument' approach was adopted, where statements about the phenomenon of interest were inferred from the selected studies (Noblit \& Hare, 1988). Our intention was to develop a line of argument about the likely response of patients to a guided selfhelp intervention.

The basic data were the main concepts reported in each of the individual studies. These concepts were synthesised across the studies to develop new ideas and interpretations. The results of this synthesis formed the basis of an explanatory framework concerning patients' experience of 
depression and its management in primary care. This framework was then applied to the specific pragmatic question driving the study: what factors might influence the effective implementation of guided self-help for depression in primary care?

\section{Stages of the meta-synthesis}

The meta-synthesis involved a number of stages:

(a) identifying the literature - topic selection, searching for the studies and appraisal of the studies;

(b) data analysis and interpretation extraction of main findings from the published studies, synthesis of main findings into an explanatory framework, application of the explanatory framework to the guided self-help intervention.

\section{Identifying the literature}

The initial search for qualitative papers in this study focused on three broad themes: studies of patients' and professionals' perspectives on help-seeking and treatment for common mental disorders in primary care; the process of implementation of self-management interventions for chronic conditions; and the use of technologies related to self-management. To identify the primary studies, the Medline, EMBASE, CINAHL and Web of Knowledge databases were searched for the period 2000-2005 inclusive. Separate search strategies were created in line with the three initial themes, with the assistance of a specialist librarian (copies of the exact search strategies are available from the authors, and an example is given as a data supplement to the online version of this paper). Twenty-four potentially relevant papers were found. Initial analysis of the papers demonstrated that the original themes used to structure the search were not clearly reflected in the available literature. Instead, studies could be grouped according to three emergent categories: patients' perspectives regarding the experience of coping with depression; patients' perspectives regarding the management of depression within primary care; and patients' attitudes towards and use of treatments commonly provided for managing depression in primary care (e.g. antidepressants and psychological therapy).

Papers were appraised using the British Sociological Association (BSA) criteria for the evaluation of qualitative research papers (BSA Medical Sociological Group, 1996). Exclusions were made if studies turned out to be insufficiently focused on the topic (e.g. not based on direct experience of depression) or if the paper was not essentially qualitative (as some studies had collected data using qualitative methods but did not analyse the data qualitatively).

\section{Data analysis and interpretation}

A grid was constructed in which each paper was entered into a separate row, and a description of the concepts derived from each paper added to the grid. The descriptions could involve the author's own words, or a paraphrase (Britten et al, 2002), in order to reliably retain the meanings and concepts of each study. The entries in this grid were then synthesised by reading the concepts and interpretations off the grid, and establishing relationships between them across the studies, in order to arrive at a broader explanatory framework. This framework was then applied to the specific issue of the delivery of guided self-help in primary care.

\section{RESULTS}

\section{Synthesis of main findings into an explanatory framework}

Nine papers were included in the metasynthesis. Elementary contextual information for the included studies is provided in a data supplement to the online version of this paper. The results of the synthesis are described below and in Table 1.

\section{Personal experience of depression}

External sources of stress or conflict were drawn upon most frequently to account for the presence of depression. These included conflict with work colleagues or family, chronic illness, events in childhood, material disadvantage and racism (Kadam et al, 2001; Rogers et al, 2001; Burr \& Chapman, 2004; Grime \& Pollock, 2004). Rather than emphasising symptoms or feelings of depression, respondents' personal experience was characterised by expressions of being unable to cope, and in particular disturbances to everyday functioning and social roles (with negative consequences for other family members) (Kadam et al, 2001; Rogers et al, 2001; Knudsen et al, 2002; Burr \& Chapman, 2004; Maxwell, 2005). Metaphors used by respondents to communicate the experience of depression included being 'on edge', 'churned-up inside', 'boxed in', 'a volcano bursting', 'broken in half', 'shut in my own little shell', 'a wall of pain' and 'prisoner in my own home' (Kadam et al, 2001). Attempts to overcome such feelings were expressed in terms such as 'fight' and 'conquer' (Kadam et al, 2001; Glasman et al, 2004).

\section{Ambivalent help-seeking and the covert presentation of psychological problems}

The experience of depression and failures to cope could lead people to seek help from formal agencies such as primary care. In relation to the decision to seek help, patients' accounts exhibited a need to leave behind more passive periods when they felt overwhelmed by feelings of their inability to cope (Rogers et al, 2001) or where inaction was leading to negative consequences for other family members, which might in turn lead to guilt (Maxwell, 2005). However, engaging with primary care services was problematic. Patients used primary care because it represented the only place where help was seen to be on offer, rather than through a specific expectation that accessing these services would be helpful. Contact with primary healthcare was relatively insignificant for the individual in the context of pressing problems and adverse circumstances that respondents reported, and patients spent very little time in face-to-face contact with their doctors or with other health professionals (Rogers et al, 2001). Accessing help was viewed as a set of 'moral actions' (Maxwell, 2005), further complicated by feelings of shame and lack of legitimacy, which could lead to the covert presentation of psychological problems (Rogers et al, 2001; Burr \& Chapman, 2004). Moreover, there was the possibility that accessing help could threaten an already weakened sense of self if it led to discussions about treatments that patients might find unacceptable (such as medication or referral to specialist mental health services). Some patients also exhibited an unquestioning attitude to the quality of care for their problems (Gask et al, 2003).

\section{Control and helplessness in engaging with treatment}

Patients reported the use of coping strategies, such as distraction or the use of particular locations associated with feelings of safety and control (Kadam et al, 2001). 
Table I Main results from the meta-synthesis

Main findings from the studies in the review

Main findings from the studies in the review

Depression related to feelings of powerlessness and lack of control, hopelessness and detachment from the world

(Burr \& Chapman, 2004)

Help sought because of a perceived inability to carry out everyday functions, loss of control, failure of previous coping strategies and impact of inaction on others (Rogers et al, 200I, 2004; Maxwell, 2005)

Use of metaphors to convey a sense of struggle (Kadam et al, 200I)

Ambiguity over the role of primary care (Kadam et al, 200I; Rogers et al, 200I)

Help-seeking from GP related to feelings of legitimacy Rogers et al, 200I; Burr \& Chapman, 2004; Maxwell, 2005)

Expectations of health services based on prior experience, and affected by feelings of shame, pessimism about effectiveness and threat (such as disruption to sense of self) (Rogers et al, 2004)

Ambivalence about need for follow-up by doctor after initial diagnosis (Gask et al, 2003)

Respondents concerned about psychological dependency, loss of autonomy and sense of failure to cope associated with use of antidepressants (Grime \& Pollock, 2004; Maxwell, 2005)

Self-help treatment enabled regained sense of self-control (Rogerset al, 2004)

Doubts of legitimacy of seeking help for depression

(Burr \& Chapman, 2004; Maxwell, 2005)

Moral dilemma caused by need to access treatment to limit impact of depression on others, but also negative beliefs about use of medication (Maxwell, 2005)

Patients exhibit low self-esteem and low expectations of care (Gask et al, 2003)

Frustration and distress caused by failure to deal with the problem, led to feelings such as weakness (Knudsen et al, 2002)

People generally not sympathetic (Kadam et al, 200I)

Threat of depression seen as continuing presence - used enactive responses and modified CBT to use independently (Glasman et al, 2004)

Medico-centred information, needs to be patient-tailored, based on individual need and experience, to help patients to self-manage (Grime \& Pollock, 2004)
Application of the explanatory framework to the guided self-help intervention
Synthesis of main findings into an explanatory framework

Personal experience of depression: depression is characterised by a sense of dissociation, loss of everyday function, and a feeling of loss of control

Ambivalent help-seeking and the covert presentation of psychological problems: patients used primary care because it happened to be there or was the only place where help was seen to be on offer.

Help-seeking is complicated by influences which result in the covert presentation of psychological problems

Control and helplessness in engagement with treatment: patients felt they had to give up control in order to engage with treatments based on biomedical principles, sense of self and usual function

Stigma associated with treatment: depression and receiving treatment for depression is stigmatised, which is related to a moral discourse about personal responsibility and the fear of loss of social function in everyday life conflicting with their desire to recover a

Incorporating personal experience in a structured intervention: materials can be sensitised to people's own experience of depression through:

(a) acknowledging the range of causal factors seen as constituting the phenomena of 'depression';

(b) using similar metaphors to express feelings associated with depression;

(c) highlighting intervention as a method of regaining control

Conceptualising the therapeutic environment for the purposes of engagement: the point at which people make contact, prior contact with sources of help and the point in their illness trajectory may be important to review in assessing the acceptability and appropriateness of guided self-help

Everyday self-management strategies and guided self-help: building on existing attempts to self-manage as part of an introduction to guided self-help may facilitate acceptance of the treatment

Managing identity and stigma: guided self-help interventions must be perceived as an acceptable way to deal with depression. This may be heightened by focusing on the degree to which the treatment can assist in regaining everyday function, and by highlighting the importance of the patient as the agent of change

Patients' understandings of self-help interventions: acceptance of self-help depends on prior experience of services

Awareness of the concept of self as the mechanism of change
Individual as change agent: professionals and their actions are ascribed greater authority and power than patients in bringing about therapeutic change. Dealing with these perceptions may be critical in any introduction to guided self-help 
Seeking out treatment was associated primarily with the perceived failure of these strategies rather than with the negative feelings or symptoms more usually associated with a diagnosis of depression (Rogers et $a l, 2001,2004)$. A key theme related to patients' feelings that they had to give up personal control over coping in order to engage with treatments based on biomedical principles such as antidepressant pharmacotherapy, which led to distinctions between 'feeling better' (due to the benefits of antidepressants) and 'being better' (a state of improved emotional well-being in the absence of medication) (Grime \& Pollock, 2004; Maxwell, 2005). Taking medication could lead to a tension between patients feeling a sense of relief because prescribed medication functioned as a prop to help them deal with difficulties in everyday life, and the need to reject such solutions as a means of taking back personal control and recovering a sense of self and social functioning (Knudsen et al, 2002; Rogers et al, 2004; Maxwell, 2005).

\section{Stigma associated with treatment}

Stigma in the context of mental health problems refers to an array of social processes focused on the personal and interpersonal aspects of creating a 'spoiled' identity (Rogers \& Pilgrim, 2005). Although it has been suggested that appeals to stigma are inadequate in explaining a reluctance to disclose emotional problems to health professionals (Prior et al, 2006), a salient theme emerging from the synthesis was the felt stigma associated with engaging with primary care. Accessing treatment for depression was not straightforward. This was partly related to feelings of a loss of control and a lack of legitimacy in accessing care for a non-physical problem (Gask et al, 2003; Burr \& Chapman, 2004), and partly because conventional treatment for depression (i.e. antidepressant medication) was associated with potential threats to the sense of self (Knudsen et al, 2002; Grime \& Pollock, 2004). In general most participants were keen to portray themselves as the type of people who do not resort to medication use, or would rather not need to resort to medication use if they could really help it (Maxwell, 2005). Taking medication was related to a moral discourse about personal responsibility, the fear of a loss of function in everyday life and a need to accept help for the sake of others (Rogers et al, 2001; Knudsen et al,
2002; Grime \& Pollock, 2004). It was only when the general practitioner or others (family or friends) offered advice to alleviate this moral dilemma were they willing to accept medication use, and even then this acceptance was contingent on the intervention being seen as short-term and temporary (Maxwell, 2005).

Respondents were unsure what to tell others about being prescribed an antidepressants and were wary of telling people that they were taking such drugs, because of the combined stigma associated with depression and the taking of antidepressants (Knudsen et al, 2002; Grime \& Pollock, 2004). The importance of change to personal identity was also raised in the studies we reviewed. Medication users reported that they felt they had become a person who needed to take antidepressants in order to get through daily life and were therefore somehow deficient. Respondents spoke of guilt and of letting themselves or others down, and expressed concerns about long-term changes to their personality associated with treatment (Grime \& Pollock, 2004).

\section{Patients' understanding of self-help interventions}

Patients' understandings of self-help interventions depend on prior experience and an awareness of the concept of self as the mechanism of change. Such awareness takes time to develop, and is difficult in the context of some of the symptoms of depression such as low self-esteem and motivation (Glasman et al, 2004; Rogers et al, 2004). The presence of a therapist offering guidance in the use of self-help materials generated ambivalence in patients about the relative role of the therapist $v$. their own use of self-help materials (Rogers et $a l, 2004)$. There is an expectation that discussions about problems are therapeutic in their own right (Kadam et al, 2001) and the development of an effective therapeutic alliance did show an impact on whether patients would subsequently use self-help (Glasman et al, 2004). Nevertheless, there was evidence of tension between the positive impact of the therapist and the negative effect on patient understanding of the therapist's role within self-help. Following contact with the therapist, patients did not always follow the principles and exercises as prescribed but reconstructed the principles of cognitive-behavioural therapy in eclectic ways that had meaning and applicability to living with psychological problems on an everyday basis. Self-help activity was described as 'hard work', and participants reported that there were times when they faced crises or lapses in their ability to use the techniques (Glasman et al, 2004).

\section{Application of the explanatory framework to the guided self-help intervention}

\section{Incorporating personal experience} in a structured intervention

People acquire an expert body of knowledge about health which includes theories about ways of managing and predicting outcomes in physical and mental health (Davison et al, 1991; Rogers \& Pilgrim, 2005), and which complements professional knowledge. This lay knowledge is concerned with people's experience of dealing with a mental health problem and its effects on social functioning, and refers to life events, their present social and psychological circumstances and their past history. In contrast, guided self-help is designed to provide patients with standardised cognitive-behavioural techniques that are known to be effective in the management of depression, which are in turn based on a psychological model of the cause of depression. Patients' descriptions of the cause of their problems differed from the psychological model, which underlies cognitive-behavioural therapy or the more biomedical notion underpinning the prescribing of antidepressants.

The metaphors used by patients in relation to depression convey a sense of struggle with thoughts and emotions, and issues of control are a dominant feature of patients' subjective experience of depression with the need to restore social functioning being prioritised over symptoms. Self-help materials, and the guidance that supports them, could use similar language and metaphors to enhance communication between patients and professionals and maximise the resources patients already bring with them. However, working effectively with people's own definitions not only involves the use of language within guided self-help materials, it also requires explicit acknowledgement of the wide range of causal factors and pathways which can account for the problems that are seen by patients as constituting the phenomena of 'depression', and active engagement with 
the broad range of explanations that lay people provide.

\section{Conceptualising the therapeutic environment for the purposes of engaging patients}

Help-seeking from primary care is typified by people waiting for long periods in a state of distress, while trying out personal coping strategies and seeking other sources of help. Despite a sense of urgency about seeking assistance when personal coping strategies fail, there is ambiguity about the role of primary care and ambivalence about its benefits. The cultural norms operating in primary care about help-seeking for a mental health problem are salient here. The synthesis pointed to the way in which people conceptualised physical problems as the 'correct' problems to be presented and managed appropriately within primary care, and this may be one of the reasons for a high rate of referral of so-called 'medically unexplained symptoms' to primary care. Although on the face of things the findings concerning the management of depression within primary care may seem tangential to the main research question concerning the optimal way of implementing guided self-help, the context of primary care and how it is perceived by patients and professionals as an arena for the disclosure and subsequent management of mental health problems is not. Receptivity, and the norms and values operating in primary care about mental health, are likely to be central in considering issues concerning the delivery of guided self-help and the promotion of primary care settings as an appropriate place from which people can seek support. Issues such as the point in the illness trajectory at which people make contact with services, and their prior contact with other sources of help, may be important to review in assessing the acceptability and appropriateness of guided selfhelp.

\section{Everyday self-management strategies and guided self-help}

The articles suggested that patients developed individual strategies for controlling feelings - although these strategies varied significantly. As noted above, guided selfhelp is designed to provide specific strategies based largely on cognitive-behavioural therapy. However, it may not be optimal simply to replace everyday strategies with those conventionally considered to be 'evidence-based'. Instead, acknowledging the importance of those everyday strategies and attempting to build on them as part of a negotiated introduction to guided self-help might encourage patient acceptance and involvement, if people were able to see the treatment as a progression from the activities that they themselves had initiated and found useful. For example, a major aspect of guided self-help is 'homework', where patients put aside time to use cognitive-behavioural techniques. Homework might be facilitated by linking to patients' existing use of specific physical locations, which provide a sense of control and safety. In this way, individual therapeutic techniques could be delivered in a way that is both 'evidence-based' and 'patient-centred'.

\section{Managing identity and stigma}

The way in which depressive problems and their management are perceived by others and affect identity emerged as prominent themes, and the extent to which the guided self-help philosophy avoids or acknowledges issues of stigma associated with medication, are likely to be key predictors of acceptability and appropriateness. If guided self-help can be discussed with individuals as a management strategy requiring a sense of acting on the world and enhancing selfworth, this might support efforts to engage people with depression by providing a means of management which is more acceptable to them and to others around them, and which allows them to avoid moral dilemmas concerning use of treatments. It may be that encouraging individuals to make a direct comparison of the benefits and drawbacks of guided self-help in contrast to those of antidepressant therapy is a means to achieve this.

\section{Individual as change agent}

Professional actions are ascribed greater authority and power than patients in bringing about therapeutic change. Generally an expected 'cultural gap' (Horowitz, 1983) exists between service users, who view their role as being in receipt of treatment rather than initiating therapy, and professionals, who are imbued with esoteric knowledge and charged with ministering to 'patients'. This representation of the facilitator in a guided self-help model as the change agent was evident in one study (Rogers et al, 2004), but other papers reported that people spoke of having the strength to overcome negative feelings and felt that depression was something that they could control. This relates to the awareness of seeing the self as the mechanism of change, which is the key to engagement with guided self-help. However, this perception was not widespread, and this may reflect a general view that treatments provided in a medical context do not require the patient to take a highly active role. There was evidence that developing the idea that the individual is the principal 'active ingredient' in guided self-help takes time, and there is a tension between the need to develop this idea and the relatively short-term nature of contact within guided self-help. It is possible that information provided before treatment begins could overcome some of the misconceptions patients might have about the nature of treatment, which could be reinforced further by contact with the therapist.

\section{DISCUSSION}

The synthesis is dependent on the particular studies included. The search was restricted to a relatively short period and might have excluded relevant studies, so the results cannot be considered definitive. The development of aspects of the explanatory framework often involved synthesising findings from only two or three studies rather than the majority; this partly reflects the fact that the research identified was fairly heterogeneous in nature. A more homogeneous set of studies might have allowed the development of a smaller number of themes, which could have been more fully described in the synthesis. The process of qualitative synthesis cannot be reduced to a set of mechanistic tasks, which raises issues of the transparency of the process. It is possible that other researchers would have synthesised the material differently. Some of the papers included in the metasynthesis were published by the present authors, and the results of this synthesis may thus be weighted towards issues identified by us in previous work.

The meta-synthesis highlighted a number of key issues that may affect the success of the introduction of guided self-help in primary care. These include the importance of issues of control and social functioning among patients with depression; the need to ensure that the context of primary care is viewed as a suitable location for mental healthcare, and supports the active role of the patient that is required in guided 
self-help; and the importance of engaging actively with patients' own constructions of depression and their current coping strategies. An interesting issue concerns potential tensions between the results of the synthesis and current professional perspectives on mental health issues. For example, cognitive-behavioural approaches to symptoms (such as exposure and behavioural activation) are designed to combat avoidance behaviour, which may clash with patient behaviours that provide a sense of control and safety.

Although the synthesis made a distinction between antidepressant therapy and guided self-help in terms of issues of control, no paper discussed the experience of patients who were using both treatments; research into the views of such patients might usefully extend understanding of issues of control in engagement with treatments, and enable services to be provided that maximise the advantages of both types of treatment.

Some of the findings of the meta-synthesis may have implications beyond the design of the guided self-help intervention. For example, the traditional biomedical view of the importance of managing depressive symptoms is challenged, as the meta-synthesis highlighted the importance of issues of control and social functioning in the decision to seek help, in the evaluation of treatments and assessment of progress. Ambivalence about taking antidepressant medication highlights the benefits of emphasising a sense of regaining control in order to engage patients with interventions. It is hoped that the initial explanatory framework developed here will serve as an impetus to further research on patient experience of mental health treatments.

\section{ACKNOWLEDGEMENTS}

The study was conducted in the context of a tria funded by the Medical Research Council (principal investigator Professor Karina Lovell), in which P.B. and A.R. are grant holders. A.R. and P.B. are funded through the Department of Health, but the views expressed in this paper are those of the authors alone. N.K. is funded by the Medical Research Council. The authors would like to thank Rosalind McNally for assistance with the search strategies, and the referees for their useful comments on the initial draft.

\section{REFERENCES}

Anderson, L., Lewis, G., Araya, R., et al (2005) Selfhelp books for depression: how can practitioners and

NAGINA KHAN, BHSc, PGC, PETER BOWER, BSc, PhD, ANNE ROGERS, BA, MSc, PhD, National Primary Care Research and Development Centre, University of Manchester, Manchester, UK

Correspondence: Nagina Khan, MRC ResearchTraining Fellow, National Primary Care Research and Development Centre, 5th Floor Williamson Building, University of Manchester, Oxford Road, Manchester MI3 9PL,UK. Email: nagina.khan@postgrad.manchester.ac.uk

(First received 6 October 2006, final revision 14 February 2007, accepted 29 March 2007)

patients make the right choice? British Journal of General Practice, 55, 387-392.

Britten, N., Campbell, R., Pope, C., et al (2002) Using meta ethnography to synthesise qualitative research: a worked example. Journal of Health Services Research and Policy, 7, 209-215.

BSA Medical Sociological Group (1996) Criteria for the evaluation of qualitative research papers. Medical Sociology News, 22, 68-7।.

Burr, J. \& Chapman, T. (2004) Contextualising experiences of depression in women from South Asian communities: a discursive approach. Sociology of Health and IIIness, 26, 433-452.

Campbell, M., Fitzpatrick, R., Haines, A., et al (2000) Framework for design and evaluation of complex interventions to improve health. BMJ, 32I, 694-696.

Campbell, R., Pound, P., Pope, C., et al (2003) Evaluating meta-ethnography: a synthesis of qualitative research on lay experiences of diabetes and diabetes care. Social Science and Medicine, 56, 671-684.

Davison, C., Davey Smith, G. \& Frankel, S. (1991) Lay epidemiology and the prevention paradox: the implications of coronary candidacy for health education. Sociology of Health and IIIness, 13, 1-19.

Department of Health (200I) Treatment Choice in Psychological Therapies and Counselling: Evidence Based Clinical Practice Guideline. UK Department of Health.

Donovan, J., Mills, N., Smith, M., et al (2002) Improving design and conduct of randomised trials by embedding them in qualitative research: ProtecT (prostate testing for cancer and treatment) study. BM] 325, 766-770.

Gask, L., Rogers, A., Oliver, D., et al (2003) Qualitative study of patients' perceptions of the quality of care for depression in general practice. British journal of General Practice, 53, 278-283.

Glasman, D., Finlay, W. \& Brock, D. (2004) Becoming a self-therapist: Using cognitive behavioural therapy for recurrent depression and/or dysthymia after completing therapy. Psychology and Psychotherapy: Therapy, Research and Practice, 77, 335-35I.

Grime, J. \& Pollock, K. (2004) Information versus experience: a comparison of an information leaflet on antidepressants with lay experience of treatment. Patient Education and Counseling, 54, 361-368.

Horowitz, A. (1983) The Social Control of Mental Illness. Academic Press.

Kadam, U., Croft, P., McLeod, J., et al (200I) A qualitative study of patients' views on anxiety and depression. British Journal of General Practice, 5I 375-380.

Knudsen, P., Hansen, E., Traulsen, J., et al (2002) Changes in self-concept while using SSRI antidepressants. Qualitative Health Research, 12 932-944.

Lovell, K., Richards, D. \& Bower, P. (2003) Improving access to primary care mental health: uncontrolled evaluation of a pilot self-help clinic. British Journal of General Practice, 53, 133-135.

Marrs, R. (1995) A meta-analysis of bibliotherapy studies. American Journal of Community Psychology, 23. 843-870.

Maxwell, M. (2005) Women's and doctors' accounts of their experiences of depression in primary care: the influence of social and moral reasoning on patients' and doctors' decisions. Chronic Illness, I, 61-7I.

Mead, N., MacDonald, W., Bower, P., et al (2005) The clinical effectiveness of guided self-help versus waiting list control in the management of anxiety and depression: a randomised controlled trial. Psychological Medicine, 35 . $1633-1643$

Medical Research Council (2000) A Framework for Development and Evaluation of RCTs for Complex Interventions to Improve Health. MRC.

Noblit, G. \& Hare, R. (1988) Meta-ethnography: Synthesising Qualitative Studies. Sage.

Prior, L., Wood, F., Lewis, G., et al (2006) Stigma revisited, disclosure of emotional problems in primary care consultations in Wales. Social Science and Medicine 56. $2191-2200$

Proudfoot, J., Ryden, C., Everitt, B., et al (2004) Clinical efficacy of computerised cognitive-behavioural therapy for anxiety and depression in primary care: randomised controlled trial. British journal of Psychiatry I85, 46-54.

Richards, D., Lovell, K. \& McEvoy, P. (2003) Access and effectiveness in psychological therapies: self-help as a routine health technology. Health and Social Care in the Community, II, 175-182.

Rogers, A. \& Pilgrim, D. (2005) Sociology of Mental Health and Illness. Oxford University Press.

Rogers, A., May, C. \& Oliver, D. (200I) Experiencin depression, experiencing the depressed: the separate worlds of patients and doctors. Journal of Mental Health, 10, 317-333.

Rogers, A., Oliver, D., Bower, P., et al (2004) People's understanding of a primary care based mental health self-help clinic. Patient Education and Counseling, 53, $4 \mid-46$.

Salkovskis, P., Rimes, K., Stephenson, D., et al (2006) A randomized controlled trial of the use of self-help materials in addition to standard general practice treatment of depression compared to standard treatment alone. Psychological Medicine, 36, 325-333.

Thorne, S., Jensen, L., Kearney, M., et al (2004) Qualitative metasynthesis: reflections on methodological orientation and ideological agenda. Qualitative Health Research, 14, 1342-1365. 